Cerebrovasc Dis 2012;33:340

DOI: $10.1159 / 000336857$

\section{Paving the Road to Translation}

\section{Nikolaus Plesnila}

Institute for Stroke and Dementia Research (ISD), University of Munich Medical School, Munich, Germany

Despite decades of significant efforts of basic researchers and clinicians, the only available causal therapeutic option for patients suffering an ischemic stroke is still thrombolysis with recombinant tissue plasminogen activator. This is a significant improvement as compared to the situation before 1995 when no specific treatment was available at all; however, since only less than $5 \%$ of all stroke patients are eligible for recombinant tissue plasminogen activator treatment, additional therapeutic options are still in urgent need. Unfortunately, so far all drug candidates found to be neuroprotective in animal models of stroke failed to produce a similar beneficial effect in patients. The cause for this 'translational roadblock' is heavily discussed and, among others, the lack of clinically relevant (large) animal models is often mentioned as one of the most important reasons.

The paper by Gauberti et al. [1] published in the current issue of Cerebrovascular Diseases addresses this problem by presenting a novel model for embolic stroke in Rhesus monkeys, one of the most widely used monkey species in experimental medicine. Following enucleation of the right eye and exposure of the distal middle cerebral artery through a craniectomy of the dorsal orbital wall, the authors used a micropipette to inject thrombin directly into the M1 branch of the middle cerebral artery. The procedure, performed under state-of-the-art anesthesia and physiological monitoring, resulted in a stable local clot, long-lasting ischemia $(>2 \mathrm{~h})$, and infarction of the cerebral cortex and striatum with no obvious vessel injury, a finding very similar to a large embolic stroke of the middle cerebral artery in humans. Neurological function was assessed by a score based on the functionality of the hand contralateral to the injured hemisphere and revealed that functional deficits persisted for at least 21 days after middle cerebral artery embolism. Dynamic changes in the ischemic brain were assessed by sequential MRI studies performed in a 3-tesla clinical MRI scanner $2 \mathrm{~h}$, and 1, 7, 21, and 90 days after middle cerebral artery occlusion. Five out of 6 animals had a significant perfusion-diffusion mismatch $2 \mathrm{~h}$ after middle cerebral artery occlusion. Infarct volume more than doubled between 2 und $24 \mathrm{~h}$ as demonstrated by diffusion-weighted imaging, thereby showing that the model produced an ischemic penumbra, i.e. tissue at risk amenable to treatment, an important feature of an experimental model developed for the evaluation of novel therapeutic strategies. The usefulness of the model is also underlined by the lack of mortality.

Although the aim and the design of the study are excellent, the model was developed according to the STAIR criteria, and the experiments were performed with an admirable level of skill and precision, the study has - like most, if not all studies performed in nonhuman primates - the limitation of using only a limited number of animals. Six Rhesus monkeys were used to establish the surgical technique and another 6 animals were used for the MRI study. However, only 3 animals were studied for more than $2 \mathrm{~h}$ since the other 3 monkeys were excluded due to an undisclosed pharmacological intervention. Accordingly, all dynamic morphological and functional changes were studied only in 3 animals, a number which makes statistical evaluations of the current results obsolete. Consequently, the authors of this truly important study are encouraged to present further data on their model, which may indeed become an important cornerstone of translational stroke research.

Taken together, the study by Gauberti et al. [1] describes a newly developed embolic occlusion model of the distal middle cerebral artery in nonhuman primates (Rhesus monkeys), which shows a dynamically growing cortical infarct within the first $24 \mathrm{~h}$ after vessel occlusion resulting in long-lasting functional deficits but no mortality. Although only 3 animals were evaluated over time, the necessary infrastructure may not be readily available to most stroke researchers, and the costs of such experiments are significant, this novel, clinically highly relevant in vivo stroke model may provide an important contribution for breaking the manacles of translational stroke research.

\section{Reference}

1 Gauberti M, Obiang P, Guedin P, Balossier A, Gakuba C, Diependaele AS, Chazalviel L, Vivien D, Young AR, Agin V, Orset C: Thrombotic stroke in the anaesthetized monkey (Macaca mulatta): characterization by MRI - a pilot study. Cerebrovasc Dis 2012;33:329-339.

Prof. Dr. med. Nikolaus Plesnila

Institute for Stroke and Dementia Research (ISD)

University of Munich Medical School, Max-Lebsche Platz 30

DE-81377 Munich (Germany)

Tel. +498970958357

E-Mail nikolaus.plesnila@med.uni-muenchen.de

\section{KARGER}

Fax +41613061234 E-Mail karger@karger.ch www.karger.com
(C) 2012 S. Karger AG, Base

$1015-9770 / 12 / 0334-0340 \$ 38.00 / 0$ 